\title{
Performance Analysis of Mobile Radio Networks with Hand-off
}

\author{
Wuyi Yue ${ }^{\dagger}$ and Yutaka Matsumoto ${ }^{\ddagger}$ \\ ${ }^{\dagger}$ Dept. of Applied Mathematics, Konan Univ., Kobe 658, Japan \\ ‡I. T. S., Inc., Kobe 658, Japan
}

\begin{abstract}
In high-capacity cellular radio communication systems, efficient channel use is achieved by covering the service area with large numbers of small cells. In such environments, a cell boundary crossing by moving users can generate many hand-off attempts. To reduce the forced termination of calls in progress, this paper considers such a priority scheme that some channels are reserved for hand-off calls and a finite buffercapacity of $N$ is offered to hand-off calls. We present an exact analysis and an efficient matrix-analytic procedure to numerically evaluate the performance of the system such as blocking probability, channel utilization, queue length and delay. Numerical results show how priority can be provided to hand-off calls according to the number of reserved channels and buffer size.
\end{abstract}

\section{Introduction}

In high-capacity cellular radio communication systems, efficient channel use is achieved by covering the service area with large numbers of small cells. However, in addition to the requirement for more fixed transceiver sites, its bottleneck is in that the small cell systems must accommodate increased numbers of cell boundary crossings of mobile users having calls in progress. The calls can include mixed voice and data transmission, image transmission, phone mail, e-mail, etc.

To enhance the quality of cellular service, hand-off priority schemes must be introduced to reduce the probability of hand-off failure. The systems with hand-off priority schemes have been studied in [1-3]. Hong and Rappaport [1] presented two prioritized hand-off procedures to the system. They assumed that the traffic is pure chance, with arrival rate $\lambda_{i}(i=1,2)$ at one cell for new and hand-off calls, negative exponentially distributed holding times with common mean $1 / \mu$. In priority scheme I, a number of channels are used exclusively for hand-off calls while the remaining channels are used for both new calls and hand-off calls. Blocked calls are cleared from the system immediately. In priority scheme II, hand-off calls can be queued in an unlimited buffer. Channels are shared in the same way as in priority scheme I. However, in micro-cell systems, the assumptions of the unlimited buffer size and the same mean holding time for both new and hand-off calls are unrealistic.

A priority queueing system modeled and analyzed in [2] is a combination of a nonpreemptive priority queueing system together with a channel reservation policy and a hysteresis mechanism. In the system it is also assumed that the channel holding time of new calls in the originating cell and the residing time of calls in the hand-off cell obeys the exponential distribution with the same mean.

As for other related studies, loss systems with two classes of calls and three groups of servers have been studied in [4-5]. Sato and Mori [4] considered a telephone-based ticket reservation system for an airline company. In the system, two streams of reservation calls are received and answered by servers. 
Each input stream is a Poisson process. The whole servers are divided into three groups. Two groups can only deal with each input stream and what they call commonly usable group is able to serve both classes of input streams. They assumed that commonly usable servers deal with streams overflowed from their own assigned servers. If servers are all busy, the newly arrived call should be lost. Kawashima [5] analyzed a similar model by approximating the overflow process to an interrupted Poisson process (IPP). He proposed an efficient and fairly accurate approximation method to compute loss probabilities for both classes of calls. It requires much shorter time than the exact method employing lumping. The models analyzed by Sato and Mori [4] and by Kawashima [5] correspond to our model without waiting room as special cases.

In this paper, we present an exact analysis to evaluate the performance of mobile radio networks with two streams of calls (referred to as new call and hand-off call) and a finite buffer-capacity of $N$ calls for hand-off. We apply a matrix-analytic method to describe the stochastic behavior of the system and we efficiently calculate the stationary probabilities with a large state space to obtain the blocking probability, channel utilization for both streams, queue length and delay for hand-off stream.

This paper is organized as follows. In Section 2, the cellular system model with hand-off is presented. Traffic performance is analyzed in Section 3 by the matrix-analytic method. In Section 4, we show numerical results and compare them with results from [4]. Conclusions are drawn in Section 5.

\section{System Model}

We consider two Poịsson streams in the system and we denote the arrival rates of new call traffic and hand-off call traffic by $\lambda_{n}$ and $\lambda_{o}$, respectively. We note that in cellular systems, $\lambda_{n}$ can be given as a system parameter, while $\lambda_{o}$ is related to other system parameters such as the arrival rate of new calls, the mean call holding time, the cell size and the users' mobility. Under memoryless assumptions, the average hand-off rate into an adjacent cell can be given by the ratio of the average call holding time to the average cell sojourn time, which is what one would intuitively expect [6]. To apply this fundamental result on mean hand-off rate in [6] to our model, we assume an independent, identically distributed cell sojourn time with mean $1 / \gamma_{n}$ for new calls and $1 / \gamma_{o}$ for hand-off calls. The holding times are assumed to be independent and exponentially distributed with different mean $1 / \mu_{n}$ for new calls and $1 / \mu_{o}$ for hand-off calls. If we consider the case of a uniform traffic distribution and at most one hand-off per call, the arrival rate of hand-off calls in a cell can be given by

$$
\lambda_{o}=\lambda_{n} \frac{\gamma_{n}}{\mu_{n}} \text {. }
$$

The total number of channels in a cell is denoted by $M$. Among $M$ channels, $R_{n}\left(R_{o}\right)$ channels are reserved for new (hand-off) calls only and the rest $\left(M-R_{n}-R_{o}\right.$ channels) are allowed to be used by both types of calls. When a call arrives, the reservation channels of its type are checked first for transmission and then the common channels. If there is no common channel available, the call is assumed to be blocked and cleared from the system if it is a new call, while the call is assumed to enter a buffer if it is a hand-off call. The buffer is assumed to have a finite capacity of $N$ calls, which are served in FIFO. If the buffer is full, however, the hand-off call will be blocked and cleared from the system. Once a call seizes a channel in a cell, it holds the channel until the transmission is completed within the cell or it moves out the cell. Whenever a call releases a channel that is a channel besides $R_{n}$, it is assumed that the first hand-off call 
waiting in the buffer, if any, immediately seizes the channel.

\section{Performance Analysis}

\subsection{State Equations}

We define the system state of a cell by the numbers of channels being used by new calls and handoff calls and the number of hand-off calls in the buffer. We formulate the model as a Markov process and observe the system at an arbitrary instant. Let $S$ denote the total number of system states for the case that the buffer is empty and $S^{\prime}$ denote the total number of system states for the case that the buffer is not empty. Then $S$ and $S^{\prime}$ are given by $S=\left[(M+1)(M+2)-R_{n}\left(R_{n}+1\right)-R_{o}\left(R_{o}+1\right)\right] / 2$, $S^{\prime}=M-R_{o}+1$. Let $x=\left(x^{(0)}, x^{(1)}, \ldots, x^{(N)}\right)$ denote the row vector of the stationary state probability distribution at an arbitrary instant, where $\boldsymbol{x}^{(i)}(0 \leq i \leq N)$ is the row vector of the stationary state probabilities for the case that there are $i$ hand-off calls in the buffer. As the size of $x^{(0)}$ is given by $S$, while the size of $x^{(i)}(1 \leq i \leq N)$ is given by $S^{\prime}$, the size of $x$ becomes $S+N S^{\prime}$. Let $i_{n}$ and $i_{o}$ denote the numbers of channels being used by new calls and hand-off calls, respectively. Given $i_{n}$ and $i_{o}$, we define a function $f\left(i_{n}, i_{o}\right)$ which maps the pair $\left(i_{n}, i_{o}\right)$ into the 1-dimensional state space $S$ as follows:

$$
f\left(i_{n}, i_{o}\right)=\left\{\begin{array}{c}
\left(M-R_{n}+1\right) i_{n}+i_{o} \\
\text { for } 0 \leq i_{n} \leq R_{n} \text { and } 0 \leq i_{o} \leq M-R_{n}, \\
(M+2) i_{n}+i_{o}-\frac{1}{2} i_{n}\left(i_{n}+1\right)-\frac{1}{2} R_{n}\left(R_{n}+1\right), \\
\text { for } R_{n}+1 \leq i_{n} \leq M-R_{o} \text { and } 0 \leq i_{o} \leq M-i_{n} .
\end{array}\right.
$$

For the case that there are waiting hand-off calls in the buffer, we also define a function $f^{\prime}\left(i_{n}, i_{o}\right)$ which maps $\left(i_{n}, i_{o}\right)$ into the 1-dimensional state space $S^{\prime}$ as follows:

$$
f^{\prime}\left(i_{n}, i_{o}\right)= \begin{cases}i_{n}, & \text { for } 0 \leq i_{n} \leq R_{n} \text { and } i_{o}=M-R_{n}, \\ i_{n}, & \text { for } R_{n}+1 \leq i_{n} \leq M-R_{o} \text { and } i_{o}=M-i_{n} .\end{cases}
$$

Using the above functions $f$ and $f^{\prime}$, we can express that $x^{(0)}=\left(x_{f(0,0)}^{(0)}, x_{f(0,1)}^{(0)}, \ldots, x_{f\left(M-R_{o}, R_{o}\right)}^{(0)}\right)$ and $\boldsymbol{x}^{(i)}=$ $\left(x_{f^{\prime}\left(0, M-R_{n}\right)}^{(i)}, x_{f^{\prime}\left(1, M-R_{n}\right)}^{(i)}, \ldots, x_{f^{\prime}\left(M-R_{o}, R_{o}\right)}^{(i)}\right), \quad(1 \leq i \leq N)$.

Let $Q$ to be the $\left(S+N S^{\prime}\right) \times\left(S+N S^{\prime}\right)$ infinitesimal matrix. $Q$ is expressed by

$$
Q=\left(\begin{array}{cccccc}
\boldsymbol{B}_{0} & \tilde{\boldsymbol{A}}_{+} & & & & 0 \\
\boldsymbol{B}_{-} & \boldsymbol{A}_{0} & \boldsymbol{A}_{+} & & & \\
& \boldsymbol{A}_{-} & \boldsymbol{A}_{0} & \boldsymbol{A}_{+} & & \\
& & \ldots & \ldots & \ldots & \\
& & & \boldsymbol{A}_{-} & \boldsymbol{A}_{0} & \boldsymbol{A}_{+} \\
0 & & & & \boldsymbol{A}_{-} & \tilde{A}_{0}
\end{array}\right) .
$$

As $x Q=0$ and $x \boldsymbol{e}=1$, we have

$$
\begin{aligned}
& x^{(0)} B_{0}+x^{(1)} B_{-}=\mathbf{o}, \\
& x^{(0)} \tilde{A}_{+}+x^{(1)} A_{0}+x^{(2)} A_{-}=\mathbf{o}, \\
& x^{(i-1)} A_{+}+x^{(i)} A_{0}+x^{(i+1)} A_{-}=\mathbf{o} \\
& x^{(N-1)} A_{+}+x^{(N)} \tilde{A}_{0}=\mathbf{o}, \\
& x^{(0)} e+\sum_{k=1}^{N} x^{(k)} e=1
\end{aligned}
$$


where $e$ is a $N$ dimensional column vector with all elements equal to 1 .

The elements of $\boldsymbol{B}_{0}, \boldsymbol{B}_{-}, \tilde{\boldsymbol{A}}_{+}, \boldsymbol{A}_{0}, \boldsymbol{A}_{+}, \boldsymbol{A}_{-}, \tilde{\boldsymbol{A}}_{0}$ are infinitesimal generators that we define as follows: $\boldsymbol{B}_{0}$ is an $S \times S$ infinitesimal submatrix that corresponds to the case that no hand-off call is in the buffer. The elements of $\boldsymbol{B}_{0}$ are given as follows:

(i) Transition rates from $i_{n}=0$ and $i_{o}=0$ are given by

$$
b_{f(0,0) f(0,1)}=\lambda_{o}, \quad b_{f(0,0) f(1,0)}=\lambda_{n}, \quad b_{f(0,0) f(0,0)}=-\left(\lambda_{o}+\lambda_{n}\right) .
$$

(ii) Transition rates from $1 \leq i_{n} \leq M-R_{o}-1$ and $i_{o}=0$ are given by

$$
\begin{aligned}
& b_{f\left(i_{n}, i_{o}\right) f\left(i_{n}, i_{o}+1\right)}=\lambda_{o}, \quad b_{f\left(i_{n}, i_{o}\right) f\left(i_{n}+1, i_{o}\right)}=\lambda_{n}, \quad b_{f\left(i_{n}, i_{o}\right) f\left(i_{n}-1, i_{o}\right)}=i_{n} \mu_{n}, \\
& b_{f\left(i_{n}, i_{o}\right) f\left(i_{n}, i_{o}\right)}=-\left(\lambda_{o}+\lambda_{n}+i_{n} \mu_{n}\right) .
\end{aligned}
$$

(iii) Transition rates from $i_{n}=M-R_{o}$ and $i_{o}=0$ are given by

$$
\begin{aligned}
& b_{f\left(i_{n}, i_{o}\right) f\left(i_{n}, i_{o}+1\right)}=\lambda_{o}, \quad b_{f\left(i_{n}, i_{o}\right) f\left(i_{n}-1, i_{o}\right)}=i_{n} \mu_{n}, \\
& b_{f\left(i_{n}, i_{o}\right) f\left(i_{n}, i_{o}\right)}=-\left(\lambda_{o}+i_{n} \mu_{n}\right) .
\end{aligned}
$$

(iv) Transition rates from $i_{n}=0$ and $1 \leq i_{o} \leq M-R_{n}-1$ are given by

$$
\begin{aligned}
& b_{f\left(i_{n}, i_{o}\right) f\left(i_{n}, i_{o}+1\right)}=\lambda_{o}, \quad b_{f\left(i_{n}, i_{o}\right) f\left(i_{n}+1, i_{o}\right)}=\lambda_{n}, \quad b_{f\left(i_{n}, i_{o}\right) f\left(i_{n}, i_{o}-1\right)}=i_{o} \mu_{o}, \\
& b_{f\left(i_{n}, i_{o}\right) f\left(i_{n}, i_{o}\right)}=-\left(\lambda_{o}+\lambda_{n}+i_{o} \mu_{o}\right) .
\end{aligned}
$$

(v) Transition rates from $i_{n}=0$ and $i_{o}=M-R_{n}$ are given by

$$
\begin{aligned}
& b_{f\left(i_{n}, i_{o}\right) f\left(i_{n}, i_{o}-1\right)}=i_{o} \mu_{o}, \quad b_{f\left(i_{n}, i_{o}\right) f\left(i_{n}+1, i_{o}\right)}=\lambda_{n}, \\
& b_{f\left(i_{n}, i_{o}\right) f\left(i_{n}, i_{o}\right)}=-\left(\lambda_{o}+\lambda_{n}+i_{o} \mu_{o}\right),
\end{aligned}
$$

where in case of $N=0$, and $i_{n}+i_{o}=M$ or $i_{o}=M-R_{n}$, then $b_{f\left(i_{n}, i_{o}\right) f\left(i_{n}, i_{o}\right)}=-\left(\lambda_{n}+i_{o} \mu_{o}\right)$.

(vi) Transition rates from $1 \leq i_{n} \leq R_{n}, \quad 1 \leq i_{o} \leq M-R_{n}-1$ and $R_{n}+1 \leq i_{n} \leq M-R_{o}-1$, $1 \leq i_{o} \leq M-i_{n}-1$ are given by

$$
\begin{aligned}
& b_{f\left(i_{n}, i_{o}\right) f\left(i_{n}, i_{o}+1\right)}=\lambda_{o}, \quad b_{f\left(i_{n}, i_{o}\right) f\left(i_{n}+1, i_{o}\right)}=\lambda_{n}, \quad b_{f\left(i_{n}, i_{o}\right) f\left(i_{n}, i_{o}-1\right)}=i_{o} \mu_{o}, \\
& b_{f\left(i_{n}, i_{o}\right) f\left(i_{n}-1, i_{o}\right)}=i_{n} \mu_{n}, \quad b_{f\left(i_{n}, i_{o}\right) f\left(i_{n}, i_{o}\right)}=-\left(\lambda_{o}+\lambda_{n}+i_{n} \mu_{n}+i_{o} \mu_{o}\right) .
\end{aligned}
$$

(vii) Transition rates from $i_{n}=M-R_{o}$ and $1 \leq i_{o} \leq R_{o}-1$ are given by

$$
\begin{aligned}
& b_{f\left(i_{n}, i_{o}\right) f\left(i_{n}, i_{o}+1\right)}=\lambda_{o}, \quad b_{f\left(i_{n}, i_{o}\right) f\left(i_{n}, i_{o}-1\right)}=i_{o} \mu_{o}, \quad b_{f\left(i_{n}, i_{o}\right) f\left(i_{n}-1, i_{o}\right)}=i_{n} \mu_{n}, \\
& b_{f\left(i_{n}, i_{o}\right) f\left(i_{n}, i_{o}\right)}=-\left(\lambda_{o}+i_{n} \mu_{n}+i_{o} \mu_{o}\right) .
\end{aligned}
$$

(viii) Transition rates from $1 \leq i_{n} \leq R_{n}-1$ and $i_{o}=M-R_{n}$ are given by

$$
\begin{aligned}
& b_{f\left(i_{n}, i_{o}\right) f\left(i_{n}+1, i_{o}\right)}=\lambda_{n}, \quad b_{f\left(i_{n}, i_{o}\right) f\left(i_{n}, i_{o}-1\right)}=i_{o} \mu_{o}, \quad b_{f\left(i_{n}, i_{o}\right) f\left(i_{n}-1, i_{o}\right)}=i_{n} \mu_{n}, \\
& b_{f\left(i_{n}, i_{o}\right) f\left(i_{n}, i_{o}\right)}=-\left(\lambda_{o}+\lambda_{n}+i_{n} \mu_{n}+i_{o} \mu_{o}\right),
\end{aligned}
$$

where in case of $N=0$, and $i_{n}+i_{o}=M$ or $i_{o}=M-R_{n}$, then $b_{f\left(i_{n}, i_{o}\right) f\left(i_{n}, i_{o}\right)}=-\left(\lambda_{n}+i_{n} \mu_{n}+i_{o} \mu_{o}\right)$. (ix) Transition rates from $R_{n} \leq i_{n} \leq M-R_{o}$ and $i_{o}=M-i_{n}$ are given by

$$
b_{f\left(i_{n}, i_{o}\right) f\left(i_{n}, i_{o}-1\right)}=i_{o} \mu_{o}, \quad b_{f\left(i_{n}, i_{o}\right) f\left(i_{n}-1, i_{o}\right)}=i_{n} \mu_{n}, \quad b_{f\left(i_{n}, i_{o}\right) f\left(i_{n}, i_{o}\right)}=-\left(\lambda_{o}+i_{o} \mu_{o}+i_{n} \mu_{n}\right),
$$


where in case of $N=0$, and $i_{n}+i_{o}=M$ or $i_{o}=M-R_{n}$, then $b_{f\left(i_{n}, i_{o}\right) f\left(i_{n}, i_{o}\right)}=-\left(i_{n} \mu_{n}+i_{o} \mu_{o}\right)$.

$\boldsymbol{B}_{-}$is an $S^{\prime} \times S$ infinitesimal submatrix which corresponds to the transitions from the case of having just one hand-off call in the buffer to the case that the call is severed by an available channel. $\tilde{A}_{+}$is an $S \times S^{\prime}$ infinitesimal submatrix. It is for the case that a hand-off call enters an empty buffer as no channel is available for the call upon arrival. $\boldsymbol{A}_{0}, \boldsymbol{A}_{-}, \boldsymbol{A}_{+}$and $\tilde{\boldsymbol{A}}_{0}$ are all $S^{\prime} \times S^{\prime}$ infinitesimal submatrices. $\boldsymbol{A}_{0}$ corresponds to the case that the number of hand-off calls in the buffer does not change. $\boldsymbol{A}_{0}$ is given as follows:

(i) Transition rates from $i_{n}=0$ and $i_{o}=M-R_{n}$ are given by

$$
a_{f^{\prime}\left(i_{n}, i_{o}\right) f^{\prime}\left(i_{n}+1, i_{o}\right)}=\lambda_{n}, \quad a_{f^{\prime}\left(i_{n}, i_{o}\right) f^{\prime}\left(i_{n}, i_{o}\right)}=-\left(\lambda_{o}+\lambda_{n}+i_{o} \mu_{o}\right),
$$

where in case of $N=1$, and $i_{n}+i_{o}=M$ or $i_{o}=M-R_{n}$, then $a_{f^{\prime}\left(i_{n}, i_{o}\right) f^{\prime}\left(i_{n}, i_{o}\right)}=-\left(\lambda_{n}+i_{o} \mu_{o}\right)$.

(ii) Transition rates from $1 \leq i_{n} \leq R_{n}-1$ and $i_{o}=M-R_{n}$ are given by

$$
\begin{aligned}
& a_{f^{\prime}\left(i_{n}, i_{o}\right) f^{\prime}\left(i_{n}+1, i_{o}\right)}=\lambda_{n}, \quad a_{f^{\prime}\left(i_{n}, i_{o}\right) f^{\prime}\left(i_{n}-1, i_{o}\right)}=i_{n} \mu_{n}, \\
& a_{f^{\prime}\left(i_{n}, i_{o}\right) f^{\prime}\left(i_{n}, i_{o}\right)}=-\left(\lambda_{o}+\lambda_{n}+i_{o} \mu_{o}+i_{n} \mu_{n}\right),
\end{aligned}
$$

where in case of $N=1$, and $i_{n}+i_{o}=M$ or $i_{o}=M-R_{n}$, then $a_{f^{\prime}\left(i_{n}, i_{o}\right) f^{\prime}\left(i_{n}, i_{o}\right)}=-\left(\lambda_{n}+i_{n} \mu_{n}+i_{o} \mu_{o}\right)$. (iii) Transition rates from $i_{n}=R_{n}$ and $i_{o}=M-R_{n}$ are given by

$$
a_{f^{\prime}\left(i_{n}, i_{o}\right) f^{\prime}\left(i_{n}-1, i_{o}\right)}=i_{n} \mu_{n}, \quad a_{f^{\prime}\left(i_{n}, i_{o}\right) f^{\prime}\left(i_{n}, i_{o}\right)}=-\left(\lambda_{o}+i_{o} \mu_{o}+i_{n} \mu_{n}\right),
$$

where in case of $N=1$, and $i_{n}+i_{o}=M$ or $i_{o}=M-R_{n}$, then $a_{f^{\prime}\left(i_{n}, i_{o}\right) f^{\prime}\left(i_{n}, i_{o}\right)}=-\left(i_{n} \mu_{n}+i_{o} \mu_{o}\right)$.

(iv) Transition rates from $R_{n}+1 \leq i_{n} \leq M-R_{o}$ and $i_{o}=M-i_{n}$ are given by

$$
a_{f^{\prime}\left(i_{n}, i_{o}\right) f^{\prime}\left(i_{n}, i_{o}\right)}=-\left(\lambda_{o}+i_{o} \mu_{o}+i_{n} \mu_{n}\right),
$$

where in case of $N=1$, and $i_{n}+i_{o}=M$ or $i_{o}=M-R_{n}$, then $a_{f^{\prime}\left(i_{n}, i_{o}\right) f^{\prime}\left(i_{n}, i_{o}\right)}=-\left(i_{n} \mu_{n}+i_{o} \mu_{o}\right)$.

$\boldsymbol{A}_{-}$and $\boldsymbol{A}_{+}$represent infinitesimal submatrices that the number of hand-off calls decrease and increase by one in the buffer, respectively. $\tilde{\boldsymbol{A}}_{0}$ includes the case that a hand-off call is blocked upon arrival because the buffer is full.

\subsection{Performance Measures}

Using the above infinitesimal generators, we can calculate steady state probabilities [3], through which we can obtain the utilization (which is defined as the average ratio of channels occupied by a type of calls to all channels except the ones reserved for the other type of calls), and the blocking probability as follows: In case of $N=0$, the utilizations of the new calls and hand-off calls are given by

$$
\begin{aligned}
U_{n}= & \frac{\sum_{i_{n}=0}^{R_{n}} \sum_{i_{o}=0}^{M-R_{n}} x_{f\left(i_{n}, i_{o}\right)}^{(0)} i_{n}+\sum_{i_{n}=R_{n}+1}^{M-R_{o}} \sum_{i_{o}=0}^{M-i_{n}} x_{f\left(i_{n}, i_{o}\right)}^{(0)} i_{n}}{M-R_{o}}, \\
U_{o}= & \frac{\sum_{i_{o}=0}^{R_{o}} \sum_{i_{n}=0}^{M-R_{o}} x_{f\left(i_{n}, i_{o}\right)}^{(0)} i_{o}+\sum_{i_{o}=R_{o}+1}^{M-R_{n}} \sum_{i_{n}=0}^{M-i_{o}} x_{f\left(i_{n}, i_{o}\right)}^{(0)} i_{o}}{M-R_{n}} .
\end{aligned}
$$


In case of $N=0$, the blocking probabilities of the new calls and hand-off calls are given by

$$
\begin{aligned}
& L_{n}=\sum_{i_{n}=R_{n}}^{M-R_{o}} x_{f\left(i_{n}, M-i_{n}\right)}^{(0)}+\sum_{i_{o}=0}^{R_{o}-1} x_{f\left(M-R_{o}, i_{o}\right)}^{(0)}, \\
& L_{o}=\sum_{i_{o}=R_{o}}^{M-R_{n}} x_{f\left(M-i_{o}, i_{o}\right)}^{(0)}+\sum_{i_{n}=0}^{R_{n}-1} x_{f\left(i_{n}, M-R_{n}\right)}^{(0)} .
\end{aligned}
$$

In case of $N \geq 1$, the utilizations of the new calls and hand-off calls are given by

$$
\begin{aligned}
U_{n}= & \frac{\sum_{i_{n}=0}^{R_{n}} \sum_{i_{o}=0}^{M-R_{n}}\left(x_{f\left(i_{n}, i_{o}\right)}^{(0)} i_{n}+\sum_{l=1}^{N} x_{f^{\prime}\left(i_{n}, i_{o}\right)}^{(l)} i_{n}\right)+\sum_{i_{n}=R_{n}+1}^{M-R_{o}} \sum_{i_{o}=0}^{M-i_{n}}\left(x_{f\left(i_{n}, i_{o}\right)}^{(0)} i_{n}+\sum_{l=1}^{N} x_{f^{\prime}\left(i_{n}, i_{o}\right)}^{(l)} i_{n}\right)}{M-R_{o}}, \\
U_{o}= & \frac{\sum_{i_{o}=0}^{R_{o}} \sum_{i_{n}=0}^{M-R_{o}}\left(x_{f\left(i_{n}, i_{o}\right)}^{(0)} i_{o}+\sum_{l=1}^{N} x_{f^{\prime}\left(i_{n}, i_{o}\right)}^{(l)} i_{o}\right)+\sum_{i_{o}=R_{o}+1}^{M-R_{n}} \sum_{i_{n}=0}^{M-i_{o}}\left(x_{f\left(i_{n}, i_{o}\right)}^{(0)} i_{o}+\sum_{l=1}^{N} x_{f^{\prime}\left(i_{n}, i_{o}\right)}^{(l)} i_{o}\right)}{M-R_{n}} .
\end{aligned}
$$

In case of $N \geq 1$, the blocking probabilities of the new calls and hand-off calls are given by

$$
\begin{aligned}
& L_{n}=\sum_{i_{n}=R_{n}}^{M-R_{o}} x_{f\left(i_{n}, M-i_{n}\right)}^{(0)}+\sum_{i_{o}=0}^{R_{o}-1} x_{f\left(M-R_{o}, i_{o}\right)}^{(0)}+\sum_{l=1}^{N}\left\{\sum_{i_{n}=R_{n}}^{M-R_{o}} x_{f^{\prime}\left(i_{n}, M-i_{n}\right)}^{(l)}+\sum_{i_{o}=0}^{R_{o}-1} x_{f^{\prime}\left(M-R_{o}, i_{o}\right)}^{(l)}\right\}, \\
& L_{o}=\boldsymbol{x}^{(N)} e
\end{aligned}
$$

where $\boldsymbol{e}$ is a column vector with all elements equal to 1 .

The blocking probability of an arbitrary call is given by

$$
L=\frac{\lambda_{n} L_{n}+\lambda_{o} L_{o}}{\lambda_{n}+\lambda_{o}}
$$

The average queue length of the hand-off calls is given by

$$
N_{q}=\sum_{i_{n}=0}^{M-R_{o}} \sum_{i_{o}=0}^{M-i_{n}} \sum_{l=1}^{N} l \cdot x_{f^{\prime}\left(i_{n}, i_{o}\right)}^{(l)} .
$$

The average waiting time of the hand-off calls is given by using $N_{q}$ as follows:

$$
E[W]=\frac{N_{q}}{\lambda_{o}\left(1-L_{o}\right)} .
$$

\section{Numerical Results}

In this section, we present numerical results to show system performance characteristics with handoff priority and compare it with the case without hand-off priority under the same system model and assumptions. We define that the total offered traffic load to a cell is $\Lambda=\lambda / \mu$ (erlangs/cell), the offered new traffic load is $\Lambda_{n}=\lambda_{n} / \mu_{n}$ (erlangs/cell) and the offered hand-off traffic load is $\Lambda_{o}=\lambda_{o} / \mu_{o}$ (erlangs/cell), where $\lambda=\lambda_{n}+\lambda_{o}, \mu=\mu_{n}+\mu_{o} . \lambda_{n}$ is given as a parameter and $\lambda_{o}$ is given by Eq.(1).

In Figs. 1 and 2, the utilizations and the blocking probabilities are depicted as functions of the total offered traffic load $\Lambda$ from 0.0 to 25.0 (erlangs/cell), where the mean of cell sojourn time is given by 
$\gamma_{n}=0.67$ adjusted to make the rate of $\lambda_{n}$ and $\lambda_{o}$ equal to [4] (a special case of our model without buffer). The total number of channels is $M=10$. We consider the cases with (i.e., $R_{n}, R_{o}>0$ ) and without (i.e., $R_{n}=R_{o}=0$ ) their own assigned channels for both streams for $N=0$ and examine how $R_{n}$ and $R_{o}$ change the utilization and blocking probability with the offered traffic load. We first calculate the utilizations and blocking probabilities with a given set of parameters $\lambda_{n}=3.0, \lambda_{o}=2.0, \mu_{n}=\mu_{o}=1.0$ for various assignment combinations of channels using a similar combination method of channels in [4]. Then we choose the sets of $R_{n}$ and $R_{o}$ in such a way that the blocking probabilities $L_{n}$ and $L_{o}$ become the closest.

In Figs. 1 and 2, we show the utilization and the blocking probability for three sets of $R_{n}$ and $R_{o}$ ( $R_{n}=R_{o}=0, R_{n}=3$ and $R_{o}=2, R_{n}=5$ and $R_{o}=3$ ). We observe that the difference of the utilizations among all channel assignment sets is smaller when $\Lambda$ is small, but as $\Lambda$ increases, the differences of the utilizations between with and without channel assignment become larger and the difference in case of $R_{n}=3$ and $R_{o}=2$ is larger than that in case of $R_{n}=5$ and $R_{o}=3$. The effect of channel assignment for new calls and hand-off calls is more significant particularly at larger traffic loads.

It is also observed that $U_{o}$ is smaller than $U_{n}$ in all cases. This is due to the fact that in this numerical example, the ratio of $\lambda_{n}$ to $\lambda_{o}$ is 1.5 if the other conditions are the same, so that new calls compete for all empty channels more often than hand-off calls.

As can be seen from Fig.2, the blocking probability $L_{o}$ for $R_{n}=5$ and $R_{o}=3$ has the largest value with all $\Lambda$, but the differences among all blocking probabilities are very small. This result is important because even if additional channels are given to hand-off calls, the utilization increases a lot with a little increase of blocking probabilities.

Figs. 3 and 4 show the utilization and blocking probability with buffer sizes $N=0,3,8$ to examine how buffer size $N$ could improve the performance of the system. We change the offered hand-off traffic load $\Lambda_{o}$ from 0.0 to 6.0 , which equals the given new traffic load $\Lambda_{n}=6.0$ for $M=10, R_{n}=4, R_{o}=3, \mu_{o}=\mu_{n}=$ 1.0. It should be noted in Fig. 3 that $U_{o}$ is lower than $U_{n}$ when $\Lambda_{o}$ is small. However $U_{o}$ increases with $\Lambda_{o}$ and in particular, the utilization $U_{o}$ for $N=8$ is the largest among the cases. The reason is that when $\Lambda_{o}$ is small and the fixed new traffic load is high at $\Lambda_{n}=6.0$, all channels except $R_{o}$ channels are maximumly used by new calls. The highest utilization of new calls is $U_{n}=0.7$ when $\Lambda_{o}=0$. The result of $U_{n}=0.7$ can be explained as follows. Because when $\Lambda_{o}=0$, the system behaves similarly to an $M / M / m(m)$ queueing system, where $m$ corresponds to $M-R_{o}$ in our model. By calculating the average number of new calls in the system for the special case of our model, we can also get the utilization $U_{n}=0.7$ for new calls. We conclude that the proposed hand-off priority scheme can increase the utilization for hand-off calls very much at the expense of slight reduction in the new call utilization by increasing the queue buffer size of hand-off calls.

Fig.4 shows the same improvement when increasing the queue buffer size of hand-off calls under the given parameters. Here we have checked the smallest blocking probability by comparing what is obtained by the Erlang $B$ formula with the smallest $L_{n}$ obtained by our analysis. The blocking probability $L_{o}$ of hand-off calls can be kept small for all offered hand-off traffic loads if $N$ is large. On the other hand, as $N$ increases, the blocking probability $L_{n}$ of new calls also increases. In particular, as both $N$ and the offered traffic load become larger, the blocking probability $L_{n}$ of new calls increases quickly to very large values. It means that if we increase the buffer size for hand-off calls too much, the expense of the new call will become larger. In this sense, the assumption of the unlimited buffer size for hand-off priority is unrealistic. 
Figs. 5 and 6 show the average queue length and average waiting time of hand-off calls, respectively, versus the total offered traffic load between 0.0 and 25.0, where the ratio of new call load to hand-off call load is 1.5 for $\mu_{o}=\mu_{n}=1.0, N=3,5,8$ and $M=3,8$. In case of $M=3$ we consider $R_{n}=R_{o}=1$, and in case of $M=8$ we consider $R_{n}=3, R_{o}=2$. Two main effects are clear in Figs.5 and 6: 1) with respect to the offered traffic load, how average queue length and waiting time change for different combinations between channels and buffer size, and 2) points where average queue length and average waiting time increase rapidly (at about offered traffic load $\Lambda=2.0$ and 7.5) depend on the channel $M$, but not on buffer size $N$.

\section{Conclusions}

In this paper, we have presented techniques for analyzing the behavior of hand-off priority schemes. We allocated the total channels to three groups: for new calls, hand-off calls and both calls, and gave hand-off calls a finite buffer-capacity of $N$. We presented a matrix-analytic solution to numerically evaluate the performance of the mobile radio network with hand-off stream, such as the blocking probability, channel utilization for both streams, queue length and delay of hand-off stream. Non-priority scheme and priority scheme are also compared in several cases and trade-offs between the performance measures of the new call and hand-off call were clarified in numerical examples.

From the above numerical results and discussions we can conclude that to reduce the forced termination of calls in progress, one of the most promising treatments is to make reservation and buffer space for handoff calls, which results in giving a priority to hand-off calls. The priority scheme we proposed in this paper is useful to improve the system performance with hand-off calls.

The matrix-analytic solution for the stationary state probability distribution derived in this paper makes it possible to further calculate the higher moments of waiting time for hand-off stream. This extension is given in [3].

\section{Acknowledgment}

The authors would like to thank The Hyogo Science and Technology Association, for supporting this research. The authors would also like to thank Master course student F.Tan of Konan University for helping the numerical calculation.

\section{References}

[1] D. Hong and S. S. Rappaport, "Traffic Model and Performance Analysis for Cellular Mobile Radio Telephone Systems with Prioritized and Nonprioritized Hand-off Procedures," IEEE Trans. on Veh. Tech., Vol.VT-35, No.3, pp.77-92, 1986.

[2] D. McMillan, "Delay Analysis of a Cellular Mobile Priority Queueing System," IEEE/ACM Trans. on Networking, Vol.3, No.3, 1995.

[3] W. Yue and Y. Matsumoto, "Probability Distribution of Delay in Cellular Mobile Networks with Hand-off," in preparation.

[4] T. Sato and M. Mori, "An Application of the Lumping Method to a Loss System with Two Types of Customers," J. of the Opera. Res. Socie. of Japan, Vol.26, No.1, pp.51-59, 1983.

[5] K. Kawashima, "An Approximation of a Loss System with Two Heterogeneous Types of Calls," J. of the Opera. Res. Socie. of Japan, Vol.28, No.2, pp.163-176, 1985.

[6] S. Nanda, "Teletraffic Models for Urban and Suburban Microcells: Cell Sizes and Hand-off Rates," IEEE Trans. on Veh. Tech., Vol.VT-42, No.4, pp.673-682, 1993. 


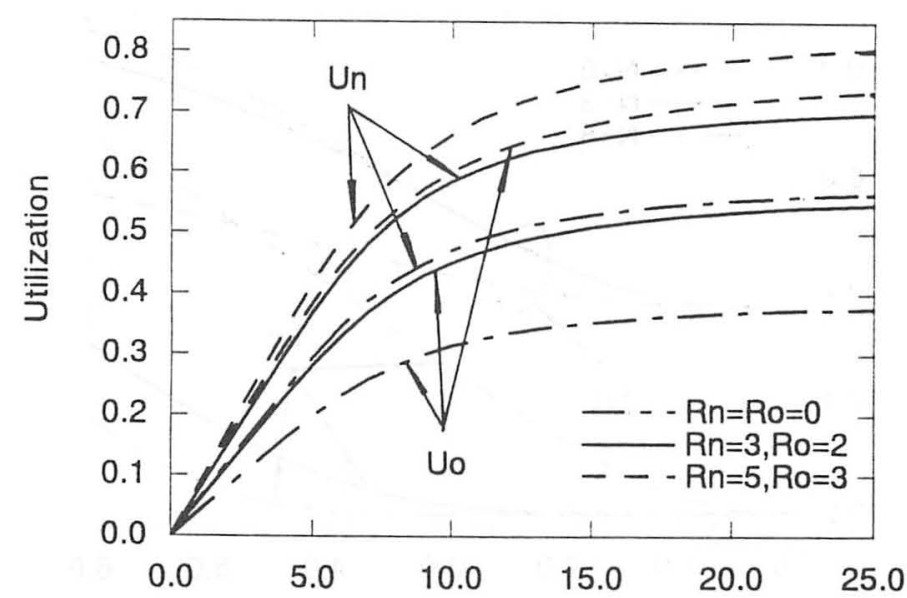

Total Offered Traffic Load $\Lambda$ (erlangs/cell)

Figure 1: Utilization vs. total offered traffic load $\Lambda$.

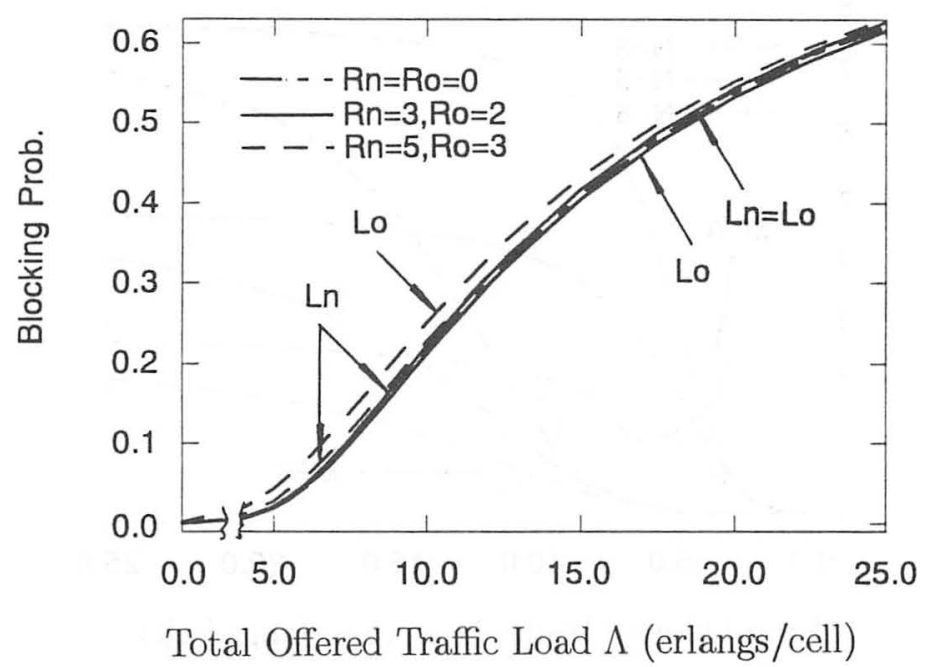

Figure 2: Blocking probability vs. total offered traffic load $\Lambda$.

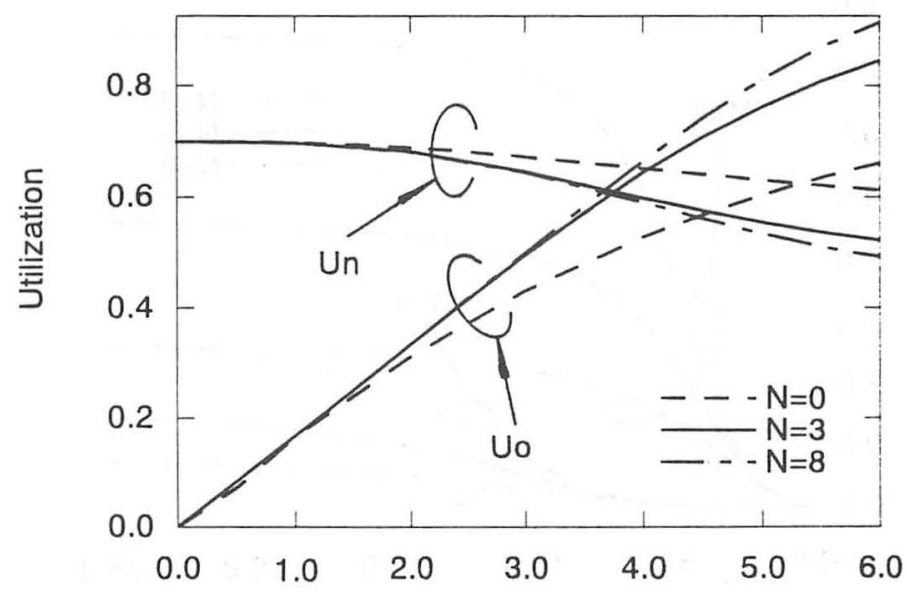

Offered Hand-off Traffic Load $\Lambda_{o}$ (erlangs/cell)

Figure 3: Utilization vs. offered hand-off traffic load $\Lambda_{o}$. 


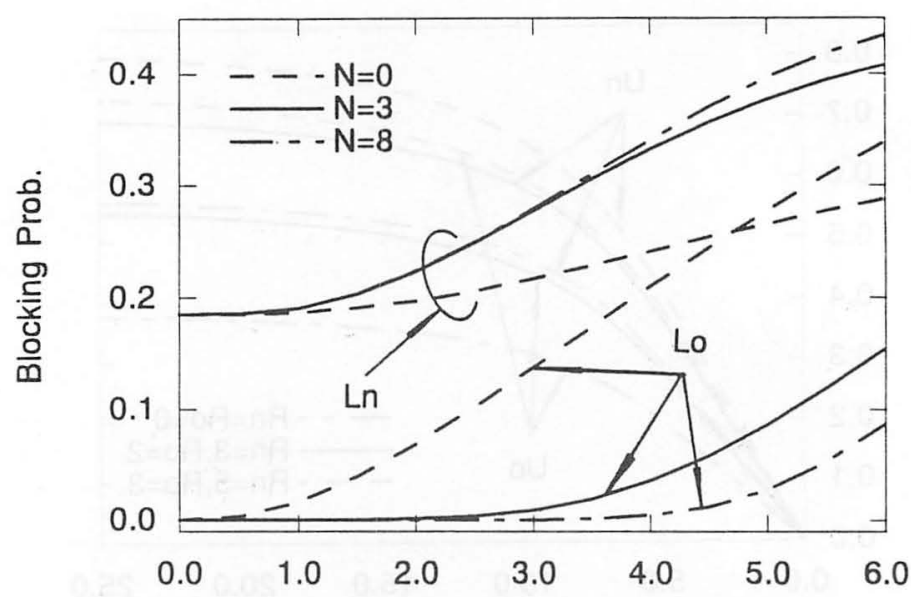

Offered Hand-off Traffic Load $\Lambda_{o}$ (erlangs/cell)

Figure 4: Blocking probability vs. offered hand-off traffic load $\Lambda_{o}$.

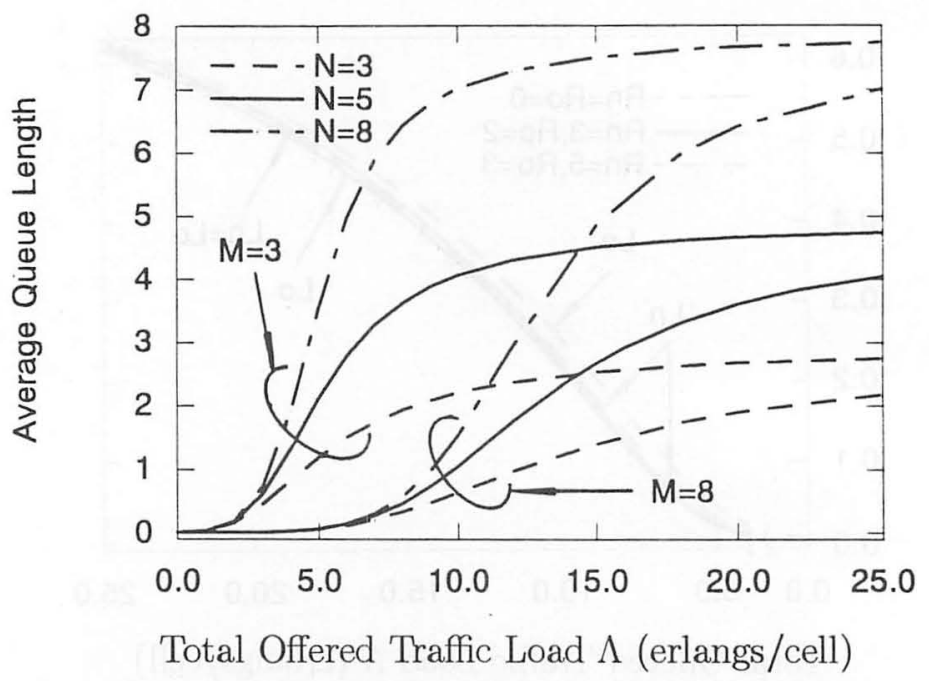

Figure 5: Average queue length vs. total offered traffic load $\Lambda$.

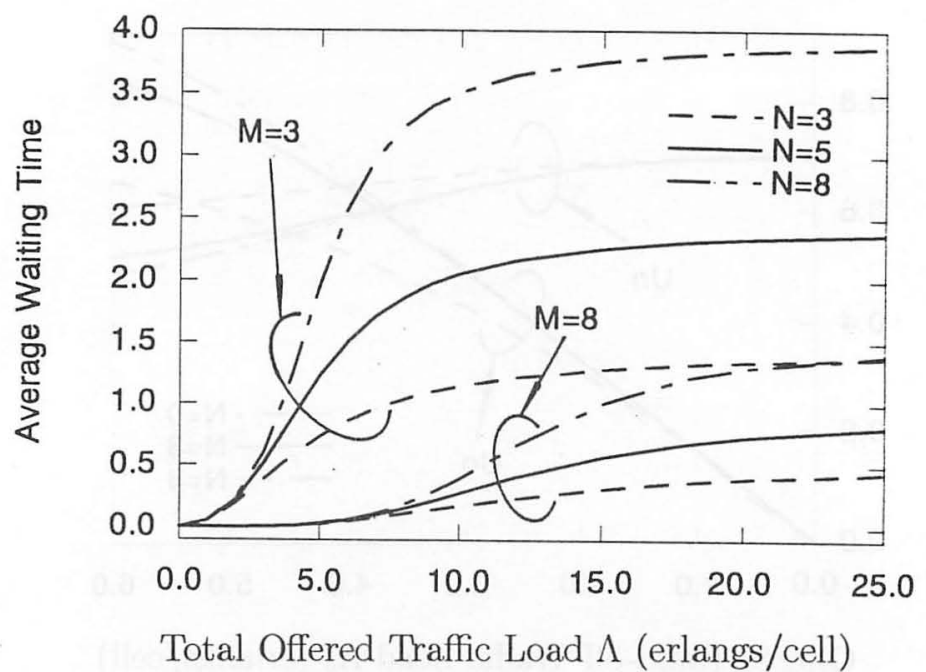

Figure 6: Average waiting time vs. total offered traffic load $\Lambda$. 